\title{
Comportamento sexual de touros zebuínos e Angus em central de coleta e processamento de sêmen
}

\author{
Daniela Sartori Rocha Bascuñan ${ }^{1}$, André Mendes Jorge ${ }^{2}$, Gilberto Pedroso da Rocha ${ }^{2}$, \\ Francisco Stefano Weschler ${ }^{2}$, Cristiana Andrighetto ${ }^{1}$
}

\footnotetext{
${ }^{1}$ Pós-Graduação em Zootecnia pela FMVZ/UNESP/Botucatu-SP

2 DPEA/FMVZ/UNESP-Botucatu-SP. Pesquisador CNPq.
}

\begin{abstract}
RESUMO - O comportamento sexual foi avaliado em 88 touros das raças Angus, Gir, Guzerá e Nelore, com 5 anos de idade, em média, peso médio de $906 \mathrm{~kg}$ e circunferência escrotal média de $41 \mathrm{~cm}$, submetidos ao manejo reprodutivo em central de coleta e processamento de sêmen. Os touros da raça Angus apresentaram menor tempo de reação se comparados aos das raças zebuínas. O tipo de estímulo manequim em movimento influenciou positivamente o tempo de reação em todas as raças estudadas. Touros mais velhos tiveram maior tempo de reação em comparação aos mais jovens. A ordem do salto no dia não influenciou o tempo de reação dos touros. Todos os eventos comportamentais dos touros Angus ocorreram próximo ao serviço completo em vagina artificial. Os eventos comportamentais cheirar e lamber genitália, golpear o manequim com a cabeça ou o chifre, gotejamento de líquido seminal, ereção e protusão de pênis e monta abortada foram realizados em maior freqüência pelos touros zebuínos que pelos Angus. Na espécie indiana, os padrões comportamentais contração prepucial e monta abortada foram mais realizados pelos touros Nelore que pelos Guzerá. O evento serviço completo sem arranque final, no entanto, foi mais observado nos Nelore e nos Guzerá que nos touros Gir.
\end{abstract}

Palavras-chave: bovinos, etologia, Gir, Guzerá, Nelore

\section{Sexual behavior of Zebu and Angus bulls at an artificial insemination center}

\begin{abstract}
This study aimed to evaluate the sexual behavior of 88 bulls of the Angus and Zebu (Gyr, Guzerat and Nellore) breeds managed for collection and processing of semen at an artificial insemination center. On average, bulls were at 5 years of age, $906 \mathrm{~kg}$ of live weight and $41 \mathrm{~cm}$ for scrotal circumference. Reaction time in all breeds was positively influenced by the "moving" stimuli teaser. Reaction time in Angus bulls was smaller than in bulls of the zebu breeds and longer in older than in younger bulls. The daily mount order did not influence the reaction time. All sexual behavior traits in the Angus bulls were close to complete mount with artificial vagina. The behavior for "smelling and licking the genitalia", "beating using the head or the horn”, "drip of seminal liquid”, "erection and penis protrusion" and "aborted mount" were more usual in zebu than in Angus bulls. Among zebu breeds "prepuce contraction” and "aborted mount” were more frequent in Nellore than in Guzerat bulls and "complete mount without end force" was more observed in Nellore and Guzerat than in Gyr bulls.
\end{abstract}

Key Words: bulls, ethology, Gyr, Guzerat, Nellore

\section{Introdução}

A produtividade de um rebanho está associada à sua eficiência reprodutiva. Contudo, apesar desta correlação, ainda existem características relacionadas à aptidão reprodutiva de touros que não foram suficientemente estudadas, principalmente nos animais da espécie Bos indicus (Fonseca et al., 1992).

Considerando a elevada representatividade do gado Zebu (Bos indicus) e seus mestiços no efetivo bovino brasileiro, de aproximadamente $80 \%$ (Costa e
Silva \& Encarnação, 1995), e o temperamento bastante nervoso e sensível ao estresse, quando esses animais são expostos a meios diferentes ao cotidiano (Crudeli et al., 1990), deve-se dar atenção especial ao estudo dessas raças.

Barbosa et al. (2001) relataram que o comportamento sexual é uma interação sócio-sexual influenciada por fatores genéticos, ambientais, nutricionais, hormonais, freqüência de acasalamentos, receptividade do heterossexo, acuidade sensorial, idade, experiência prévia do indivíduo e ordem de dominância social. 
O estudo do comportamento sexual de touros tem prestado grandes contribuições à Zootecnia, pois fornece subsídios e parâmetros de grande relevância para avaliação da libido e da capacidade de serviço dos touros. A inseminação artificial é uma das técnicas que possibilitam grandes avanços no melhoramento genético do rebanho a prazos relativamente curtos. No entanto, é imprescindível o conhecimento do comportamento sexual para determinação da fertilidade das fêmeas e dos touros e detecção do cio e da eficiência da inseminação artificial (Costa e Silva \& Encarnação, 1995; Ribeiro, 1999).

A etologia aplicada é uma ciência em expansão que tem proporcionado significativos aportes ao manejo e bemestar dos animais de produção; seu principal desafio é introduzir uma visão integrada nos sistemas de produção animal visando conciliar as técnicas de manejo aos padrões de comportamento dos animais, muitas vezes desconsiderados pelos pesquisadores.

Considerando que, em centrais de coleta a e processamento de sêmen (CCPS), os touros são mantidos fora de seu contexto sexual natural, o manejo deve ser realizado de forma ainda mais cautelosa para evitar que fatores estressantes influenciem negativamente o desempenho dos animais e reflitam em tentativas de fuga.

Esta pesquisa de etologia aplicada foi realizada visando à obtenção de resultados para quaisquer estudos futuros de estratégias de manejo na busca de possíveis soluções para o problema do condicionamento do zebuíno ao salto. Foram montados etogramas do comportamento sexual de touros de quatro raças (Nelore, Guzerá, Gir e Aberdeen Angus) para comparação destas raças quanto à freqüência dos eventos comportamentais dos touros e ao tempo de reação, considerando a idade do animal, o tipo de estímulo manequim utilizado e a ordem do salto no dia.

\section{Material e Métodos}

O projeto foi realizado na Central de Colheita e Processamento de Sêmen (CCPS) da Angus Bela Vista Pecuária Ltda, localizada no município de Pardinho (23 0,7' 46,2" de latitude e $48^{\circ} 19^{\prime} 51,0^{\prime \prime}$ de longitude), no centro-oeste do estado de São Paulo, a 959 m de altitude. A temperatura e umidade relativas médias registradas durante os 63 dias de observação foram de $25,5^{\circ} \mathrm{C}$ e $63,3 \%$, respectivamente.

Foram utilizados 88 touros adultos, com 5 anos de idade, 906 kg, 41 cm de circunferência escrotal e 10,9 coletas por touro, dados médios (Tabela 1). A distribuição dos touros por grupo genético foi realizada de acordo com suas faixas etárias (Tabelas 2 e 3).
Dos animais utilizados, 74 pertenciam à subespécie Bos indicus e 14 à subespécie Bos taurus. Todos os animais passaram por um sistema de manejo de quarentenário independentemente dos piquetes e da área de coleta e processamento de sêmen, onde foram mantidos por no mínimo 28 dias (estabelecido segundo Instrução Normativa $n^{\circ}$. 42, junho de 2003, do Ministério de Agricultura), durante o qual foram submetidos a exames sanitários e cuidados necessários para que todos atendessem aos regulamentos e às certificações de qualidade da CCPS Angus Bela Vista (ISO 9001 e ISO 14001).

Durante o manejo de rotina no quarentenário, os touros não foram submetidos a nenhum procedimento de

Tabela 1 - Distribuição dos touros observados segundo o grupo genético e número de coletas por touro por grupo genético

\begin{tabular}{lccccc}
\hline $\begin{array}{l}\text { Grupo } \\
\text { genético }\end{array}$ & Número & $\begin{array}{c}\text { Idade } \\
(\text { anos })\end{array}$ & $\begin{array}{c}\text { Peso } \\
(\mathrm{kg})\end{array}$ & $\begin{array}{c}\text { Circunferência } \\
\text { escrotal }\end{array}(\mathrm{cm})$ & $\begin{array}{c}\text { Coletas/ } \\
\text { touro }\end{array}$ \\
\hline Angus & 14 & $4 \pm 2$ & $914 \pm 120$ & $44 \pm 2$ & $11,3 \pm 9,74$ \\
Gir & 10 & $7 \pm 4$ & $702 \pm 133$ & $39 \pm 4$ & $6,9 \pm 6,23$ \\
Guzerá & 19 & $4 \pm 2$ & $799 \pm 171$ & $41 \pm 4$ & $9,7 \pm 4,86$ \\
Nelore & 45 & $6 \pm 3$ & $965 \pm 143$ & $41 \pm 3$ & $12,2 \pm 11,80$ \\
Total & 88 & $5 \pm 3$ & $906 \pm 168$ & $41 \pm 3$ & $10,9 \pm 9,83$ \\
\hline
\end{tabular}

Tabela 2 - Distribuição dos touros por grupo genético de acordo com a idade

\begin{tabular}{lcccc}
\hline Idade (anos) & \multicolumn{4}{c}{ Número de touros por grupo genético } \\
\cline { 2 - 5 } & Angus & Gir & Guzerá & Nelore \\
\hline 2 & 1 & 3 & 3 & 4 \\
3 & 5 & 1 & 6 & 8 \\
4 & 3 & 3 & 2 & 9 \\
5 & 0 & 0 & 1 & 5 \\
6 & 2 & 0 & 3 & 5 \\
7 & 2 & 0 & 1 & 3 \\
8 & 1 & 0 & 1 & 5 \\
9 & 0 & 0 & 2 & 0 \\
10 & 0 & 1 & 0 & 1 \\
11 & 0 & 1 & 0 & 1 \\
12 & 0 & 1 & 0 & 2 \\
14 & 0 & 0 & 0 & 1 \\
15 & 0 & 0 & 0 & 1 \\
\hline
\end{tabular}

Tabela 3 - Tempo médio de permanência dos touros em coleta na CCPS

\begin{tabular}{lcc}
\hline Grupo genético & Número & Tempo em coleta (meses) \\
\hline Angus & 14 & $13,0 \pm 11,83$ \\
Gir & 10 & $8,8 \pm 14,11$ \\
Guzerá & 19 & $4,3 \pm 5,65$ \\
Nelore & 45 & $6,7 \pm 8,18$ \\
Total & 88 & $7,4 \pm 9,46$
\end{tabular}


condicionamento ao salto. Os touros tiveram contato com manequins, vacas fora de estro e macho castrado mocho, apenas durante o exame andrológico e a coleta do sêmen em vagina artificial.

Depois que foram liberados para coleta de sêmen, os touros foram transferidos para piquetes individuais de capim-braquiária (Brachiaria decumbens Stapf), com água à vontade e sombra à disposição, localizados na CCPS, onde foram submetidos ao sistema de manejo.

A alimentação, composta de ração concentrada contendo $14 \%$ de proteína bruta (PB) e volumoso à base de feno de coastcross (Cynodon dactylon), foi fornecida entre 9 e $10 \mathrm{~h}$ e entre $10 \mathrm{e} 15 \mathrm{~h}$, sempre por duas pessoas distintas da equipe coletora de sêmen.

Os touros foram divididos em dois grupos para coleta de sêmen em dois dias da semana, com intervalo de dois dias para descanso. Assim, os animais cujo sêmen era coletado na segunda-feira passaram por coleta na quintafeira e aqueles cujo sêmen era colhido na terça-feira foram submetidos a nova coleta na sexta-feira. Os manequins consistiram de dez vacas fora de estro (três mestiças de cor preta, duas Aberdeen Angus pretas, três Red Angus vermelhas e duas Gir brancas) e um macho mestiço mocho castrado de cor preta. Durante o processo de coleta, foram utilizados manequins fêmeas e machos, porém, nem sempre todos os touros foram submetidos à presença de todos os manequins.

Todos os manequins foram mantidos na CCPS em um grande piquete coletivo, com capim-braquiária (Brachiaria decumbens Stapf), água à vontade, sombra e sob o mesmo manejo nutricional dos touros. O manejo de preparo para coleta consistiu no deslocamento de todos os animais (touros e manequins) de seus piquetes para o local da coleta, onde passavam por banho de água clorada, lavagem de prepúcio (touros selecionados para coleta de sêmen) e colocação de cabresto.

As vaginas artificiais foram lavadas e mantidas em estufa à temperatura ideal de $49^{\circ} \mathrm{C}\left(50^{\circ} \mathrm{C}\right.$ no calor e $60^{\circ} \mathrm{C}$ no frio) para que, no momento da coleta, se encontrassem em temperatura de 42 a $43^{\circ} \mathrm{C}$. Em seguida, foram lubrificadas com KY gel à base de água $\left(\right.$ Johnson $\left.^{\circledR}\right)$. Em cada uma delas, fixou-se um tubo de ensaio para depósito do ejaculado. Finalmente, a vagina artificial foi revestida de um cone de feltro para cobrir o cone de látex e o frasco de coleta e proteger o sêmen da radiação solar ultravioleta (UV).

A coleta foi realizada por três duplas de coletores (sempre que possível, os mesmos para os mesmos animais), entre 7h20 e 12h30. Cada touro, em seu dia de coleta, normalmente passou por dois saltos intercalados com período mínimo de descanso de 20 minutos, ou seja, duas coletas de sêmen por touro por dia. Durante o procedimento de coleta, foram mensuradas a temperatura ambiente (máxima, mínima e no momento da coleta), com termômetro de máxima e mínima Incoterm ${ }^{\circledR}$, e a umidade relativa do ar, com higrômetro seco-úmido Incoterm ${ }^{\circledR}$. O horário (em minutos) do início e término dos eventos de comportamento sexual, desde o primeiro contato físico do touro com o manequim até o serviço completo em vagina artificial (tempo de reação) foi medido com relógio digital.

Durante o processo de coleta de sêmen, registrou-se a freqüência do número médio dos seguintes eventos ou ocorrências comportamentais por touro: aproximar pelo posterior ou lateral do manequim (APL); cheirar e lamber genitália (CLG) - inspeção da genitália externa, parte interna das coxas e dos flancos, com utilização de narinas, lábios e língua; reflexo de flehmen, com ou sem inspeção de urina liberada pelo manequim (RF); cheirar e/ou lamber outras partes do corpo do manequim (COC); golpear ou tocar o manequim com a cabeça ou o chifre (GM); apoio de ganacha (APG); contração prepucial (CP); gotejamento de líquido seminal (GLS); ereção e protusão de pênis (EPP); tentativa de monta (TM); monta incompleta ou abortada (MA); serviço completo sem arranque final (SCG); serviço completo com arranque final (SCF); salto ou monta verdadeira.

Verificou-se se o manequim utilizado como estímulo estava amarrado (imobilizado) ou em movimento (manequim conduzido por um manejador que o fazia caminhar em linha reta ou em círculos, depois parando para que o touro o seguisse e se estimulasse ao salto) e a ordem do salto no dia (1으 ou $2 \underline{o}$ ).

É importante ressaltar que este estudo foi desenvolvido dentro da rotina diária de manejo da CCPS, de modo que todas as observações foram adaptadas a este contexto. Todos os registros foram realizados pela mesma pessoa.

Os resultados referentes ao tempo de reação (minutos) foram analisados por máxima verossimilhança restrita, por meio do procedimento Mixed do SAS (2001). No modelo estatístico, foram considerados os efeitos fixos de grupo genético, tipo de manequim (imobilizado ou em movimento), ordem do salto no dia (primeiro ou segundo), e as interações grupo genético $\times$ tipo de manequim e grupo genético $x$ ordem. Como efeitos aleatórios foram considerados touro dentro de grupo genético, manequim, operador e data de observação e, como covariável, foi incluído o efeito linear de idade.

Os efeitos de grupo genético foram desdobrados mediante os seguintes contrastes linearmente independentes, de acordo com a espécie (Bos taurus ou Bos indicus) e 
dentro da espécie zebuína de acordo com o temperamento (mais agitado, Nelore e Guzerá, ou mais tranqüilo, Gir): Angus vs Zebu; Nelore e Guzerá vs Gir; e Nelore vs Guzerá.

Os dados foram analisados considerando-se como nível de significância $\mathrm{P} \leq 0,05$ e probabilidade de erro tipo I.

Os resultados referentes às 13 variáveis comportamentais foram analisados pelo mesmo procedimento, porém, não se incluiu efeito de idade no modelo estatístico, pois este efeito não foi significativo $(\mathrm{P}>0,05)$.

\section{Resultados e Discussão}

O tempo de reação dos touros Angus (Tabela 4) foi menor que o dos touros zebuínos $(\mathrm{P}<0,05)$, que não diferiram entre si $(\mathrm{P}>0,05)$. Estes resultados confirmam os obtidos em investigações anteriores de que, em comparação a animais europeus, os touros zebuínos são mais lentos em reagir a fêmeas em estro, o que indica a existência de diferenças de ordem genética no comportamento sexual desses animais (Chenoweth et al., 1996; Pineda et al., 1997). Barbosa et al. (1991) demonstraram que touros da raça Canchim apresentam menor tempo de reação se comparados a touros da raça Nelore.

Uma vez que neste estudo todos os manequins utilizados se encontravam fora de estro, os resultados obtidos contrariam os descritos por Anderson (1948, 1949), citado por Hafez (1975), de que touros de raças européias montam rapidamente vacas fora de estro ou outros touros, enquanto os de raças zebuínas apresentam comportamento sexual caracteristicamente lento e montam apenas vacas em estro.

Por outro lado, resultados semelhantes foram reportados por Rao et al. (1990) em estudo realizado em central de coleta e processamento de sêmen na Índia. Segundo esses autores, com vacas fora de estro, touros da raça Zebu são sexualmente mais lentos que os europeus.

Existe um consenso entre pesquisadores de que são necessários mais estudos para elucidar a predisposição do touro zebuíno ao salto. O tempo de reação gasto com manequim em movimento foi menor (Tabela $5, \mathrm{P}<0,05$ ) e

Tabela 4 - Tempo de reação, em minutos, de acordo com o grupo genético

\begin{tabular}{lcc}
\hline Grupo genético & Tempo de reação (minutos) & Contraste \\
\hline Angus & $3,9 \pm 2,42$ & $1: \mathrm{P}=0,001$ \\
Gir & $12,2 \pm 3,02$ & $2: \mathrm{P}=0,774$ \\
Guzerá & $12,0 \pm 1,89$ & $3: \mathrm{P}=0,321$ \\
Nelore & $14,1 \pm 1,26$ & \\
\hline
\end{tabular}

* 1: Angus vs Zebu; 2: Nelore e Guzerá vs. Gir e 3: Nelore vs Guzerá (probabilidade de erro tipo I). CV (a) $=34,97 \% ; C V(b)=64,66 \%$. sugere que este estímulo foi mais eficiente em desencadear a cadeia de respostas comportamentais nos touros.

Não houve efeito de interação grupo genético $\times$ tipo de manequim ( $\mathrm{P}=0,129)$. De acordo com Hafez (1975), a estimulação visual oferecida ao touro estimula a realização de seus padrões de comportamento sexual. Além disso, o touro tem preferência por determinadas situações de estímulo e essas preferências podem ser facilmente estabelecidas pelo condicionamento ou aprendizado associativo, o que indica pouca evidência de origem inata.

Os resultados corroboram os relatos de Wierzbowski \& Janasz (1965), citados por Hafez (1975), de que qualquer objeto tridimensional semelhante ao posterior de uma vaca poderia atuar como estímulo sexual e que o movimento do objeto aumenta o efeito estimulativo. O conjunto físico completo também tem influência no estímulo visual do touro, portanto, para estimular os animais, é importante oferecer ambientes ou contextos sexuais distintos (Hafez, 1975).

Neste contexto, é desejável em um ambiente de CCPS estabelecer estímulos condicionais associados a melhores respostas sexuais dos touros, por meio do manejo mais adequado e cauteloso do ambiente.

Um touro saciado em relação a um estímulo animal em um ambiente particular freqüentemente responderá positivamente se lhe for oferecido um novo estímulo animal ou se o mesmo estímulo animal lhe for apresentado em um novo ambiente ou contexto físico (Almquist \& Hale 1956; citados por Hafez, 1975; Hale, 1966; Price, 1987).

Hale \& Almquist (1960), citados por Price (1987), observaram que, localizando o manequim animal em um novo local, houve redução de 75 a 85\% no tempo de reação dos touros, portanto, a saciedade sexual é, em parte, uma resposta psicológica a um contexto estímulo-específico.

Touros também são estimulados sexualmente ao assistirem outros touros saltando, assim como quando assistidos por outros (Mader \& Price, 1984, citados por Geary \& Reeves, 1992). Esses tipos de estímulo visual estiveram presentes na CCPS.

A idade do touro aumentou o tempo de reação (Figura 1). Resultados diferentes foram descritos por Barbosa et al.

Tabela 5 - Tempo de reação, em minutos, de acordo com o tipo de manequim

\begin{tabular}{lcc}
\hline Tipo de manequim & Tempo de reação (minutos) & $\mathrm{P} *$ \\
\hline Imobilizado & $12,6 \pm 1,74$ & 0,012 \\
Em movimento & $8,5 \pm 1,08$ & \\
\hline
\end{tabular}

* Probabilidade de erro tipo I. CV $(a)=34,97 \% ; C V(b)=64,66 \%$. 
(1991), que comprovaram que o tempo de reação diminuiu com o aumento da idade dos touros, de 27 para 39 meses, e que essa diferença poderia ser atribuída à maturidade sexual, ainda incompleta aos 27 meses de idade. No entanto, a variação de idade utilizada por esses autores foi diferente da apresentada neste estudo.

Apesar da maior faixa etária dos animais zebuínos estudados, não foi considerada a idade do touro de acordo com seu grupo genético, pois a interação idade $\times$ grupo genético não foi significativa $(\mathrm{P}>0,05)$. Uma vez eliminados os touros de faixa etária extrema, de 14 e 15 anos de idade, a tendência linear observada permaneceu.

Acuña et al. (1988) relataram que, em testes de capacidade de serviço, uma vez alcançada a maturidade sexual, touros mais velhos poderiam apresentar padrões de excitação mais lentos, diminuição da libido e aumento de patologias de pênis e artropatias. Como os animais deste estudo se encontravam saudáveis, livres de qualquer enfermidade e fora de seus ambientes naturais, o maior tempo de reação pode ser atribuído ao alto mérito genético e à elevada demanda desde muito cedo em suas vidas. Assim, deve-se atentar ao manejo desses indivíduos no intuito de desvendar quais pressões de estímulo seriam mais apropriadas nesses casos.

A ordem do salto no dia não afetou o tempo de reação despendido pelos touros ( $P>0,05$, Tabela 6 ). Não houve efeito da interação grupo genético $\times$ ordem do salto do touro por dia $(\mathrm{P}=0,314)$. Segundo Hafez (1975), a natureza do fenômeno de recuperação (período refratário) a partir da saciedade de um estímulo sexual não é bem entendida. O tempo necessário para um touro se recuperar de uma saciedade a um conjunto de estímulo particular tem grande variabilidade individual. Assim, um touro que se recupera rapidamente mostrará tempo de reação constante durante

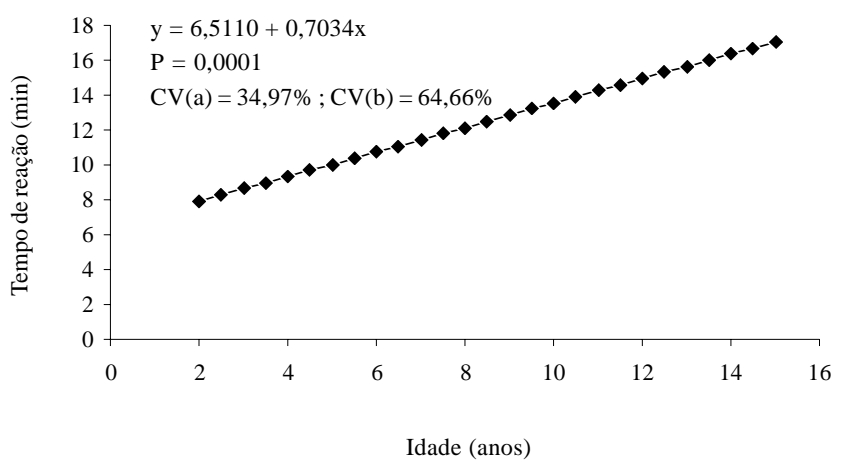

Figura 1 - Tempo de reação de acordo com a idade do touro, independentemente do grupo genético.
Tabela 6 - Tempo de reação, em minutos, de acordo com a ordem do salto no dia

\begin{tabular}{lcc}
\hline Ordem do salto & Tempo de reação (minutos) & P* \\
\hline Primeiro & $10,3 \pm 1,25$ & 0,632 \\
Segundo & $10,8 \pm 1,31$ &
\end{tabular}

* Probabilidade de erro tipo I. CV(a) $=34,97 \%$; CV $(b)=64,66 \%$

sucessivas provas semanais perante as mesmas situações de estímulo.

Crombach (1961) e Signoret (1962), citados por Hafez (1975) reportaram que a estimulação extra, um período de descanso e a permissão de falsas montas antes da coleta de sêmen aumentam a excitação sexual e melhoram a qualidade do ejaculado.

Os resultados das médias das ocorrências (eventos por touro) de cada uma das atitudes comportamentais expressas pelos touros (Tabela 7) indicaram diferenças $(\mathrm{P}<0,05)$ em alguns dos eventos comportamentais:

- cheirar e lamber genitália (CLG) - evento comportamental expresso em maior intensidade pelos touros zebuínos (contraste $1, \mathrm{P}=0,042$ );

- golpear o manequim com a cabeça ou o chifre (GM) - padrão comportamental realizado com maior freqüência pelos zebuínos que pelos taurinos (contraste 1 ; $\mathrm{P}=0,036$ );

- contração prepucial (CP) - diferença observada apenas entre touros indianos; evento mais realizado pelos touros da raça Nelore que por aqueles da raça Guzerá (contraste 3; $\mathrm{P}=0$,009);

- gotejamento de líquido seminal (GLS) - houve diferença entre as duas espécies observadas; evento mais realizado pelos zebuínos (contraste $1 ; \mathrm{P}=0,003$ );

- ereção e protusão do pênis (EPP) - evento expresso com maior freqüência pelos touros zebuínos que pelos taurinos (contraste 1 ; $\mathrm{P}=0,022$ );

- monta abortada (MA) - diferença entre as duas espécies (contraste $1 ; \mathrm{P}=0,003$ ). Este padrão comportamental foi observado mais nos animais da raça Zebu e, dentro da espécie indiana, foi mais realizado pelos touros Nelore que pelos Guzerá (contraste 3; P = 0,036);

- serviço completo sem arranque final (SCSAF) diferença observada apenas dentro da espécie indiana. Esse evento foi mais realizado pelos touros Nelore e Guzerá que pelos Gir (contraste 2; $\mathrm{P}=0,011$ ).

Costa e Silva (2002) reportou que, em condições de campo ou em testes de libido, o padrão comportamental tentativa de monta em touro europeu, em comparação ao Nelore, ocorreu em freqüência bem mais próxima à do serviço completo. 
Tabela 7 - Ocorrências (evento/touro) de cada comportamento sexual

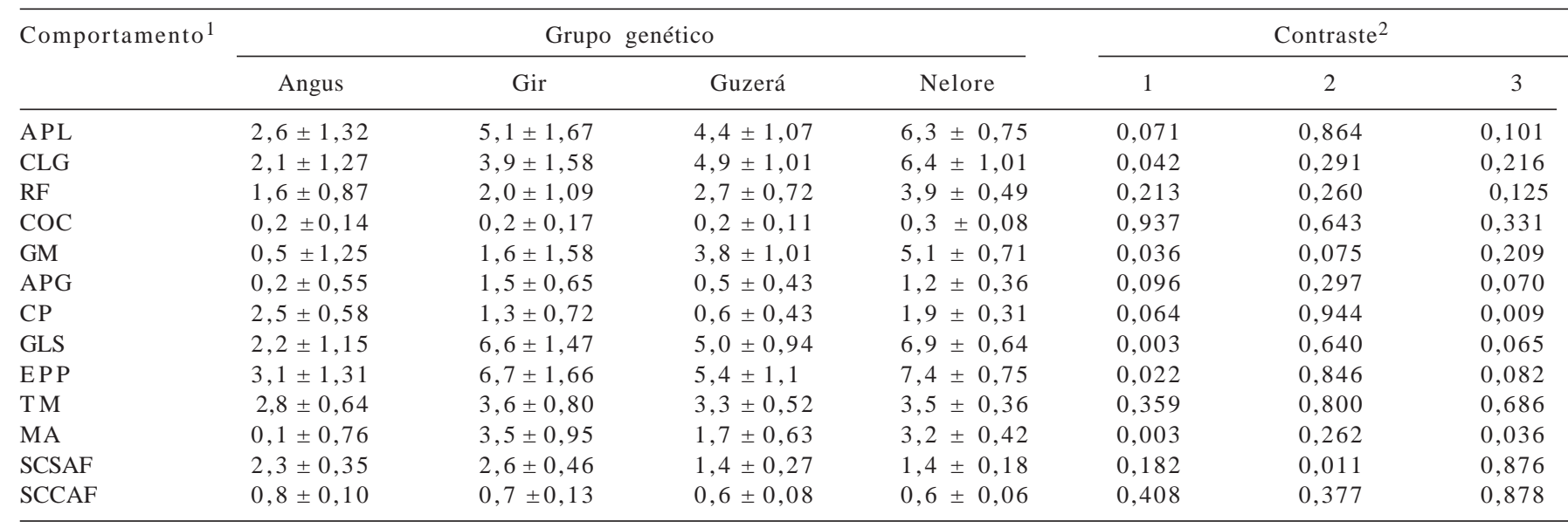

${ }^{1}$ APL - aproximação pelo posterior ou lateral do manequim; CLG - cheirar e lamber genitália externa; RF - reflexo de flehmen com ou sem inspeção de urina; COC - cheirar e/ou lamber outras partes do manequim; GM - golpear suavemente com a cabeça ou o chifre; APG - apoio de ganacha; CP - contração prepucial; GLS - gotejamento de líquido seminal; EPP - ereção e protusão de pênis; TM - tentativa de monta; MA - monta abortada; SCG - serviço completo sem arranque final; SCF - serviço completo com arranque final.

21: Angus vs Zebu; 2: Nelore e Guzerá vs Gir e 3: Nelore vs Guzerá (probabilidade de erro tipo I).

Os resultados deste estudo confirmam esse fato em ambiente de CCPS, demonstrando que o tempo de reação do Bos taurus é menor que o de Bos indicus (Tabela 4), de modo que todos os eventos comportamentais estudados nos touros europeus ocorreram mais próximo do serviço completo com ejaculação em vagina artificial (Tabela 7). Esse fato sugere que os touros Angus foram mais efetivos em montar que os zebuínos.

Os touros Zebuínos, quando comparados aos europeus $(\mathrm{P}<0,05)$, expressaram mais os eventos comportamentais cheirar e lamber genitália, golpear o manequim com a cabeça ou o chifre, gotejamento de líquido seminal, ereção e protusão de pênis e monta abortada, sugerindo que os touros Bos indicus, neste ambiente específico, são mais cortejadores que os europeus.

Dentro da espécie indiana, os eventos comportamentais contração prepucial e monta abortada foram mais realizados pelos touros Nelore que pelos Guzerá $(\mathrm{P}<0,05)$. O evento serviço completo sem arranque final, no entanto, foi mais observado nos Nelore e nos Guzerá em comparação aos $\operatorname{Gir}(\mathrm{P}<0,05)$, o que indica diferenças comportamentais quantitativas entre raças de touros pertencentes à mesma espécie.

Costa e Silva (2002), em estudo realizado no Pantanal Sul-Mato-Grossense, relatou que os comportamentos de cortejo na espécie Bos pantaindicus foram mais demorados e envolventes e resultaram em menor número de montas, fato também reportado por Chenoweth (1993) e Vale Filho (1980), citados por Costa e Silva (1995).

Maiores estudos devem ser realizados visando elucidar os padrões de comportamento sexual em touros Zebu para que técnicas de manejo mais apropriadas possibilitem melhor estimulação e predisposição ao salto.

\section{Conclusões}

Apesar do maior tempo de reação dos zebuínos submetidos ao ambiente de central de coleta e processamento de sêmen, é possível diminuir o tempo de reação desses touros por meio do estímulo manequim em movimento. Touros mais velhos apresentam tempo de reação mais longo em comparação aos mais jovens, portanto, devem ser manejados com maior atenção e cautela. Touros Angus são mais eficientes na expressão dos eventos comportamentais estudados em ambiente de central de coleta e processamento de sêmen. No entanto, os zebuínos são mais cortejadores. É altamente desejável a realização de mais estudos comportamentais em centrais de coleta e processamento de sêmen para observação dos estímulos condicionais associados a respostas sexuais mais positivas em touros nessas condições de manejo reprodutivo.

\section{Literatura Citada}

ALLER, J.F.; ALBERIO, R.H. La ganadería bovina argentina y el uso de biotecnologías en la reproducción. Cabia, v.30, p.2427, 1996

ACUÑA, C.M.; APELlAniZ, A.; CANOSA, M.R. Prueba de capacidad de servicio en toros de rodeos de cria en la cuenca del salado. Revista Argentina de Reproducción Animal, v.8, n.6, p.523-529, 1988.

BARBOSA, R.T.; ALENCAR, M.N.; BARBOSA, P.F. et al. Comportamento sexual de touros das raças Canchim e Nelore. Revista Brasileira de Reprodução Animal, v.15, n.4-3, p.151-154, 1991. 
CHENOWETH, P.J.; CHASE JR., C.C.; LARSEN, R.E. et al. The assesment of sexual performance in young Bos Taurus and Bos indicus beef bulls. Applied Animal Behaviour Science, v.48, p.225-236, 1996.

COSTA E SILVA, E.V.; ENCARNAÇÃO, R. O comportamento sexual e manejo reprodutivo de bovinos de corte. In: ENCONTRO ANUAL DE ETOLOGIA, 13., 1995, Pirassununga. Anais... Pirassununga: Sociedade Brasileira de Etologia, 1995. p. $70-82$

COSTA E SILVA, E.V.; SERENO, J.R.B.; PARANHOS DA COSTA et al. Comportamento sexual de touros Nelore (Bos indicus) e Pantaneiro (Bos taurus) durante os procedimentos de teste de libido. Revista Brasileira de Reprodução Animal, v.23, n.3, p.214-216, 1999.

COSTA E SILVA, E.V. Comportamento sexual de touros Nelore (Bos Taurus indicus) em monta a campo e em testes de libido. Jaboticabal: Universidade Estadual Paulista, 2002. 137p. Tese (Doutorado em Zootecnia) - Universidade Estadual Paulista, 2002.

CRUDELI, G.A.; FONSECA, V.O.; COSTA E SILVA, E.V. et al. Comportamiento sexual de toros Nelore (Bos taurus indicus): efecto de la capacidad de servicio sobre la tasa de fertilidad del rodeo. Cabia, n.21, p.20-26, 1990.

FRASER, A.F. The influence of psychological and other factors on reation time in the bull. Cornell Vet, v.50, n.1, p.126-132, 1960.
FONSECA, V.O.; CRUDELI, G.A.; COSTA E SILVA, E.V. et al. Aptidão reprodutiva de touros da raça Nelore. Efeito das diferentes estações do ano sobre as características seminais, circunferência escrotal e fertilidade. Arquivo Brasileiro de Medicina Veterinária e Zootecnia, v.44, n.1, p.7-75, 1993.

GEARY, T.W.; REEVES, J.J. Relative importance of vision and olfaction for detection of estrus by bulls. Journal of Animal Science, v.70, n.9, p.2726-2731, 1992.

HAFEZ, E.S.E.; BOUISSOU, M.F. The behaviour of cattle. In: HAFEZ, E.S.E. (Ed.). The behaviour of domestic animals 3.ed. London: Bailliére Tindall, 1975. p.203-245.

HALE, E.B. Visual stimuli and reproductive behavior in bulls Journal of Animal Science, v.25, p.36-48, 1966 (suppl. 1)

PINEDA, N.; LEMOS, P.F.; FONSECA, V.O. et al. Comparação entre dois testes de avaliação do comportamento sexual (libido) de touros Nelore (Bos taurus indicus). Revista Brasileira de Reprodução Animal, v.21, n.4, p.29-34, 1997.

PRICE, E.O. Male sexual behavior. The Veterinary Clinics of North América - Food Animal Practice, v.3, n.2, p.405-422, 1987.

RIBEIRO, W.N. Comportamento sexual de bovinos. In: CONGRESSO DE ETOLOGIA, 17., 1999, Botucatu. Anais... Botucatu: Sociedade Brasileira de Etologia, 1999. (CD-ROM).

NARASIMHA RAO, A.V.; SREEMANARAYANA, O.; VISWESWARA RAO, C. Studies on sex behaviour and seminal traits in Ongole, Jersey, Jersey x Ongole and Murrah bulls. Indian Veterinary Journal, p.284-287, 1990. 